\title{
Dose-escalated intensity-modulated radiotherapy and irradiation of subventricular zones in relation to tumor control outcomes of patients with glioblastoma multiforme
}

\author{
This article was published in the following Dove Press journal: \\ OncoTargets and Therapy \\ 2 March 2016 \\ Number of times this article has been viewed
}

\author{
Grace Kusumawidjaja' \\ Patricia Zhun Hong Gan' \\ Whee Sze Ong ${ }^{2}$ \\ Achiraya Teyateeti ${ }^{3}$ \\ Pittaya Dankulchai ${ }^{3}$ \\ Daniel Yat Harn Tan' \\ Eu Tiong Chua' \\ Kevin Lee Min Chua' \\ Chee Kian Tham ${ }^{4}$ \\ Fuh Yong Wong' \\ Melvin Lee Kiang Chual,5 \\ 'Division of Radiation Oncology, \\ National Cancer Centre, Singapore; \\ ${ }^{2}$ Division of Clinical Trials and \\ Epidemiological Sciences, National \\ Cancer Centre, Singapore; \\ ${ }^{3}$ Department of Radiology, Division \\ of Radiation Oncology, Faculty of \\ Medicine Siriraj Hospital, Mahidol \\ University, Thailand; ${ }^{4}$ Division of \\ Medical Oncology, National Cancer \\ Centre, Singapore; ${ }^{5}$ Duke-NUS \\ Graduate Medical School, Singapore
}

Correspondence: Melvin Lee Kiang Chua Division of Radiation Oncology, National Cancer Centre, II Hospital Drive,

Singapore 169610

$\mathrm{Tel}+6563214204$

$\mathrm{Fax}+6562234854$

Email melvin.chua.I.k@nccs.com.sg

\begin{abstract}
Background: Glioblastoma multiforme (GBM) is the most aggressive primary brain tumor with high relapse rate. In this study, we aimed to determine if dose-escalated (DE) radiotherapy improved tumor control and survival in GBM patients.

Methods: We conducted a retrospective analysis of 49 and 23 newly-diagnosed histologyproven GBM patients, treated with DE radiotherapy delivered in 70 Gy (2.33 Gy per fraction) and conventional doses (60 Gy), respectively, between 2007 and 2013. Clinical target volumes for 70 and 60 Gy were defined by 0.5 and $2.0 \mathrm{~cm}$ expansion of magnetic resonance imaging T1-gadolinium-enhanced tumor/surgical cavity, respectively. Bilateral subventricular zones (SVZ) were contoured on a co-registered pre-treatment magnetic resonance imaging and planning computed tomography dataset as a $5 \mathrm{~mm}$ wide structure along the lateral margins of the lateral ventricles. Survival outcomes of both cohorts were compared using log-rank test. Radiation dose to SVZ in the DE cohort was evaluated.

Results: Median follow-up was 13.6 and 15.1 months for the DE- and conventionally-treated cohorts, respectively. Median overall survival (OS) of patients who received DE radiotherapy was 15.2 months $(95 \%$ confidence interval $[\mathrm{CI}]=11.0-18.6)$, while median OS of the latter cohort was 18.4 months $(95 \% \mathrm{CI}=12.5-31.4, P=0.253)$. Univariate analyses of clinical and dosimetric parameters among the DE cohort demonstrated a trend of longer progression-free survival, but not OS, with incremental radiation doses to the ipsilateral SVZ (hazard ratio $[\mathrm{HR}]=0.95,95 \% \mathrm{CI}=0.90-1.00, P=0.052$ ) and proportion of ipsilateral $\mathrm{SVZ}$ receiving $50 \mathrm{~Gy}$ ( $\mathrm{HR}=0.98,95 \% \mathrm{CI}=0.97-1.00, P=0.017$ ).
\end{abstract}

Conclusion: DE radiotherapy did not improve survival in patients with GBM. Incorporation of ipsilateral SVZ as a radiotherapy target volume for patients with GBM requires prospective validation.

Keywords: glioblastoma multiforme, intensity-modulated radiotherapy, dose escalation, subventricular zones

\section{Background}

Glioblastoma multiforme (GBM) represents the commonest primary brain tumor, with an estimated annual incidence of 2-3 cases per 100,000 people. In patients diagnosed with the disease, few survive beyond 2 years, even with combination temozolomide (TMZ) and radiotherapy, although stratification based on MGMT promoter methylation status identifies individuals who will benefit most from this manner of treatment intensification. ${ }^{1,2}$ Nonetheless, in patients lacking the methylated epigenome, alternative 
systemic agents as radiation-sensitizers have not proved to be superior to TMZ. ${ }^{3-7}$ Assessment of recurrences in the era of patients treated with TMZ and radiotherapy continues to indicate a predominant pattern of central relapses, thus hinting at the necessity for other measures to intensify local treatment. ${ }^{8}$ From the mechanistic perspective, a postulation for the distinct resistance of GBM to local therapy relates to the constant clonal evolution of neural stem cells into tumor precursors that may be intrinsically radiation resistant. ${ }^{9,10}$ Failure to completely eradicate these primordial tumor clones would ultimately lead to a recurrence. In support of this notion, a number of series have now reported that GBM lesions that exist in close proximity to the anatomical location where neural stem cells exist, namely the subventricular zones (SVZ), are associated with an aggressive phenotype. ${ }^{11,12}$

In this context, we retrospectively investigated if radiotherapy dose escalation of 70 Gy to the gross disease gave a better tumor control and survival in GBM patients. Given the implementation of higher than conventional radiotherapy doses in this study, we further explored the association of radiation dose to the $\mathrm{SVZ}$ and clinical outcomes.

\section{Methods}

\section{Patients}

We analyzed case records of 49 patients with newly-diagnosed and histologically proven GBM, who were treated with a dose-escalated (DE) radiotherapy regime at the National Cancer Centre Singapore between 2007 and 2013. Due to the modest number of conventionally-treated patients at the National Cancer Centre Singapore, we collaborated with Siriraj hospital, Thailand, to review records of 23 patients with the same eligibility criteria, who were treated with a conventional dose of 60 Gy over the same time period. Patients with multi-focal disease and/or a history of previous head and neck radiotherapy, as well as unavailability of posttreatment magnetic resonance imaging (MRI) scans were excluded from the analysis.

Ethical approval for the study was obtained from Singhealth Centralised Institutional Review Board (reference number 2013/658/B) and Siriraj Institutional Review Board (reference number Si086/2015). The requirement for informed consent of patients was waived by each institutional review board due to the retrospective nature of this study. Patient records/information was anonymized and de-identified prior to analysis.

\section{Radiotherapy}

The DE treatment scheme, designed on the basis of an $\alpha / \beta$ estimate of 2 Gy for late neurological adverse effects, involved delivering an additional $10 \mathrm{~Gy}$ boost simultaneously using intensity-modulated radiotherapy, resulting in a cumulative dose of 70 Gy over 30 fractions (equivalent 2 Gy dose $\left[\mathrm{EQD}_{2}\right]=71.9 \mathrm{~Gy}, \alpha / \beta_{\text {tumor }}=10 \mathrm{~Gy} ; 75.8 \mathrm{~Gy}$, $\left.\alpha / \beta_{\text {late tissues }}=2 \mathrm{~Gy}\right)$. The following target volumes were defined. Fused MRI and computed tomography (CT) images, obtained at $3 \mathrm{~mm}$ slice thickness, were utilized for target volume delineation. Gadolinium-enhanced lesions observed on MRI T1 sequence were contoured as gross tumor volume (GTV). Clinical target volume $\left(\mathrm{CTV}_{60}\right)$ was defined as a 2.0 $\mathrm{cm}$ margin around the GTV, encompassing MRI T2 postoperative enhancing perilesional area, as well as taking into account anatomical barriers. The boost volume $\left(\mathrm{CTV}_{70}\right)$ was defined as a $5 \mathrm{~mm}$ circumferential expansion of the GTV. ${ }^{13}$

In the conventionally-treated cohort, patients received 60 Gy delivered as a single phase treatment. CTV ${ }_{60}$ was defined as above.

Treatment-related toxicity was graded according to Common Terminology Criteria for Adverse Events version 4.0. Advanced imaging (magnetic resonance spectroscopy) and surgical resection for radiation necrosis were not mandated in the treatment protocol, and were ordered at the discretion of the individual clinician.

\section{Chemotherapy}

Concurrent and adjuvant TMZ were administered at doses of 75 and $150-200 \mathrm{mg} / \mathrm{m}^{2} /$ day, respectively. Concurrent TMZ was delivered daily for 6 weeks, and in the adjuvant phase, it commenced a month post-radiation and continued for a total of six cycles, each cycle lasting 5 days, repeated every 28 days. Prophylaxis against Pneumocystis jiroveci pneumonia was given when TMZ was used concurrently with radiotherapy.

\section{Follow-up and assessment of patterns of relapses}

Follow-up MRI scan was done at 2- to 4-monthly intervals. Progression was determined based on T1 post-contrast MRI in axial as well as coronal planes. We relied on the Response Assessment in Neuro-Oncology criteria ${ }^{14}$ to differentiate between true progression and pseudoprogression. T1 postgadolinium MRI brain, which defined recurrence of disease, were imported were imported to the Eclipse ${ }^{\mathrm{TM}}$ treatment planning system (Varian Medical Systems, Palo Alto, CA, USA) and fused retrospectively with the planning CT images. Following fusion verification, the recurrence was contoured on the planning CT. Structures representing the $60 \mathrm{~Gy}$ and $70 \mathrm{~Gy}$ isodose lines were derived and Boolean operators were used to obtain the volume of recurrence within each isodose 
line. Ratio of overlapping to total recurrence volumes were then derived for each isodose, and patterns of relapses classified as such, central ( $>95 \%$ overlap), in-field ( $>80 \%-95 \%)$, marginal (20\%-80\%), and distal $(<20 \%)$.

\section{Evaluation of radiation dosimetry in SVZ}

Bilateral SVZ were contoured on a fused pre-treatment MRI and planning CT dataset as a $5 \mathrm{~mm}$ wide structure along the lateral margins of the lateral ventricles ${ }^{15}$ (Figure 1). Volume receiving $50 \mathrm{~Gy}\left(\mathrm{~V}_{50}\right)$ and mean dose received were then recorded for ipsilateral, contralateral, and composite SVZ structures.
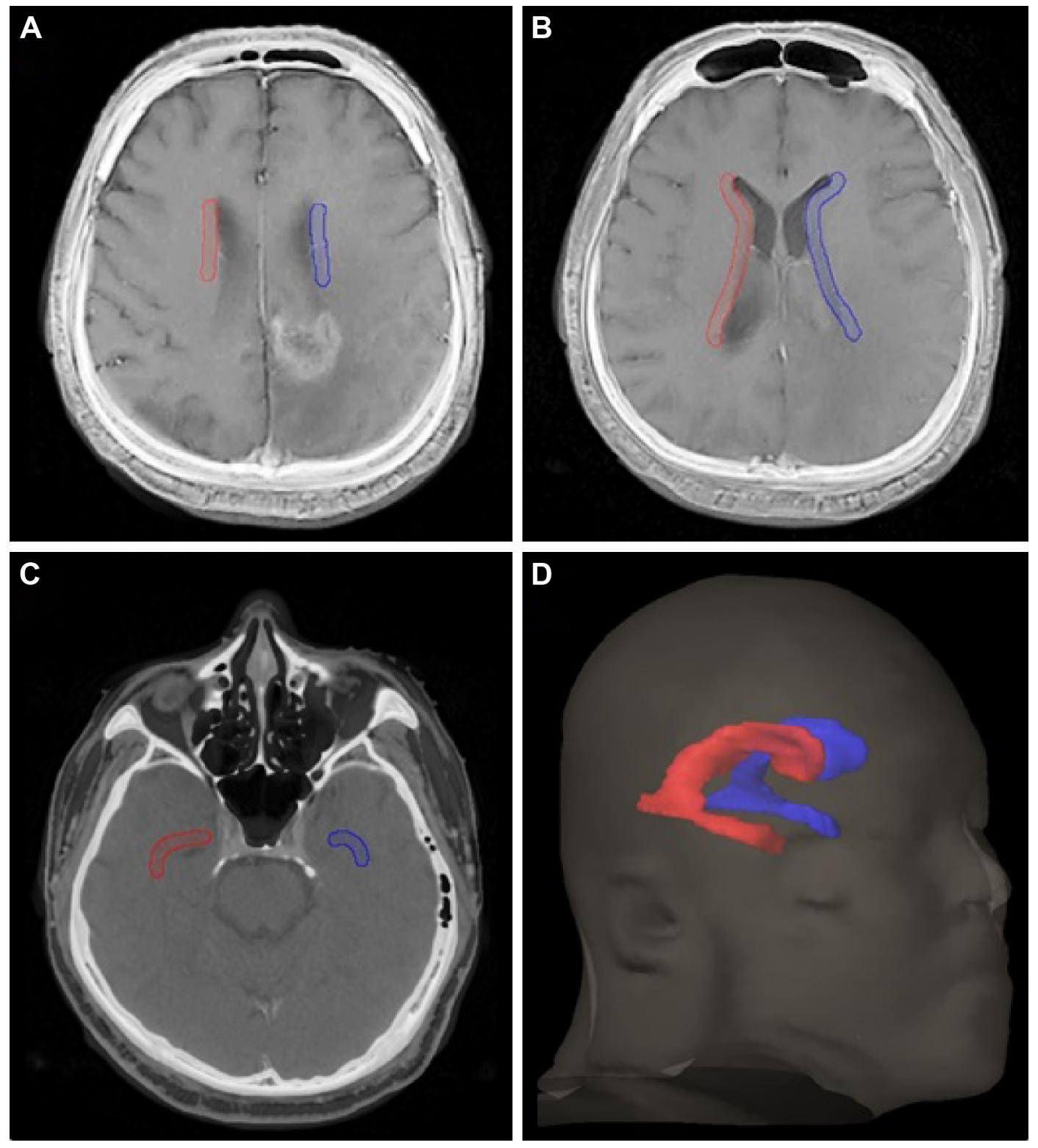

Figure I Bilateral subventricular zones (SVZ) contours.

Notes: Representative images of bilateral subventricular zones' (SVZ) contours on superior (A), middle (B), and inferior (C) slices of planning computed tomography (CT) dataset; (D) 3-dimensional reconstructed image. 
at the date of their last follow-up. Survival distributions were estimated using the Kaplan-Meier method, and compared based on the log-rank test for categorical characteristics and the Wald test for continuous characteristics. Cox proportional hazards regression models were fitted to examine the association of clinical and treatment parameters with PFS and OS. Variables with $P$-value $<0.05$ in the univariate analysis were selected for the multivariate Cox proportional hazards model. The proportional hazards assumption was verified using Schoenfeld's residuals. All analyses were performed using SAS version 9.4 (SAS Institute Inc., Cary, NC, USA). Statistical significance was set as a threshold of $P$-value $<0.05$.

\section{Results}

\section{Patient and treatment characteristics}

Median age at diagnosis was 54 and 53 years old for the DE- and conventionally-treated cohorts, respectively. Patient and treatment characteristics of the two cohorts were largely similar, with the exception of performance status (Table 1). In particular, 43 of 49 patients $(87.8 \%)$ in the DE cohort and 19 of $23(82.6 \%)$ patients in the conventional cohort received TMZ with radiotherapy.

\section{Clinical outcomes}

Median follow-up of all patients in the DE cohort was 13.6 months (range 1.7-66.2). There were eight patients alive at the time of analysis, and their median follow-up was 28.7 months. Only one patient in the DE cohort was histologically proven to have radiation necrosis. This particular patient survived for $\geq 4$ years. Three other patients survived for $\geq 3$ years, and five for $\geq 2$ years.

In the conventional cohort, median follow-up was 15.1 months (range 5.3-63.6). There were six patients alive at the time of analysis, and their median follow-up was 14.1 months. Overall, no grade 3 or 4 toxicity in either cohort was observed throughout the treatment course. Median OS was 15.2 months (95\% confidence interval $[\mathrm{CI}]=11.0-18.6$ ) for DE cohort and 18.4 months (95\% CI $=12.5-31.4$ ) for conventionally-treated patients, respectively, corresponding to a hazard ratio $(\mathrm{HR})$ of $0.72(95 \% \mathrm{CI}=0.41-1.27 ; P=0.253$, Figure 2). Median PFS for the DE and conventionally-treated cohorts was 7.1 months $(95 \% \mathrm{CI}=5.6-9.6)$ and 11.1 months (95\% CI $=6.0-24.6)$, respectively, corresponding to an HR of 0.57 (95\% CI $=0.32-0.99 ; P=0.043$, Figure 2 ).

Univariate Cox regression analysis among DE-treated cohort was performed to identify independent significant determinants of prognosis. Among the tested variables, receipt of TMZ and performance status were associated with OS and multivariate analysis confirmed that they were independent prognosticators. Patients with Eastern Cooperative Oncology Group performance status of $2-3(\mathrm{HR}=2.37 ; 95 \% \mathrm{CI}=1.06-5.32 ; P=0.036)$ and TMZ non-recipients (HR $=2.51 ; 95 \% \mathrm{CI}=1.00-6.27$; $P=0.049)$ were associated with an inferior OS.

\section{Patterns of relapses}

Within the $60 \mathrm{~Gy}$ isodose, 12 relapses $(70.6 \%)$ occurred to be central and one in-field (5.9\%) in the conventional cohort,

Table I Patient and treatment characteristics

\begin{tabular}{|c|c|c|c|c|c|c|c|}
\hline & \multicolumn{2}{|l|}{ Total } & \multicolumn{2}{|c|}{$\begin{array}{l}\text { Dose-escalated } \\
\text { cohort }\end{array}$} & \multicolumn{2}{|c|}{$\begin{array}{l}\text { Conventional } \\
\text { cohort }\end{array}$} & \multirow[t]{2}{*}{$P$-value } \\
\hline & Number & $\%$ & Number & $\%$ & Number & $\%$ & \\
\hline Total & 72 & 100 & 49 & 100 & 23 & 100 & \\
\hline \multicolumn{8}{|l|}{ Age at diagnosis, years } \\
\hline Median (range) & $53.5(|6-8|)$ & & $54(|6-8|)$ & & $53(3|-7|)$ & & 0.814 \\
\hline \multicolumn{8}{|l|}{ Sex } \\
\hline Male & 43 & 59.7 & 30 & 61.2 & 13 & 56.5 & 0.704 \\
\hline Female & 29 & 40.3 & 19 & 38.8 & 10 & 43.5 & \\
\hline \multicolumn{8}{|l|}{ Type of surgery } \\
\hline Biopsy & 13 & |8.| & 10 & 20.4 & 3 & 13.0 & 0.215 \\
\hline Partial resection & 30 & 41.7 & 17 & 34.7 & 13 & 56.5 & \\
\hline Gross total resection & 29 & 40.3 & 22 & 44.9 & 7 & 30.4 & \\
\hline \multicolumn{8}{|c|}{ Eastern Cooperative Oncology Group performance status } \\
\hline $0-1$ & 62 & 86.1 & 39 & 79.6 & 23 & 100 & $0.02 *$ \\
\hline $2-3$ & 10 & 13.9 & 10 & 20.4 & 0 & 0 & \\
\hline \multicolumn{8}{|l|}{ Temozolomide } \\
\hline Yes & 62 & 86.1 & 43 & 87.8 & 19 & 82.6 & 0.716 \\
\hline No & 10 & 13.9 & 6 & 12.2 & 4 & 17.4 & \\
\hline
\end{tabular}

Note: *Statistically significant difference between dose-escalated and conventionally treated cohorts. 
A

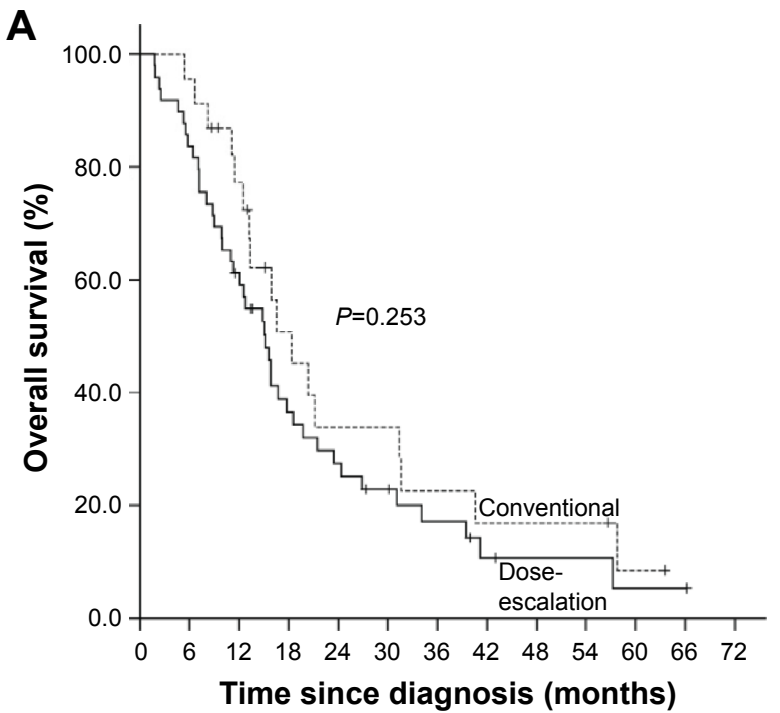

Number at risk:

$$
\text { Dose- }
$$

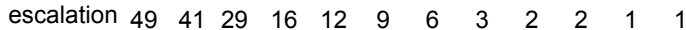

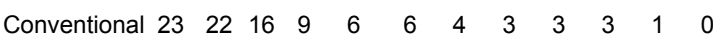

B

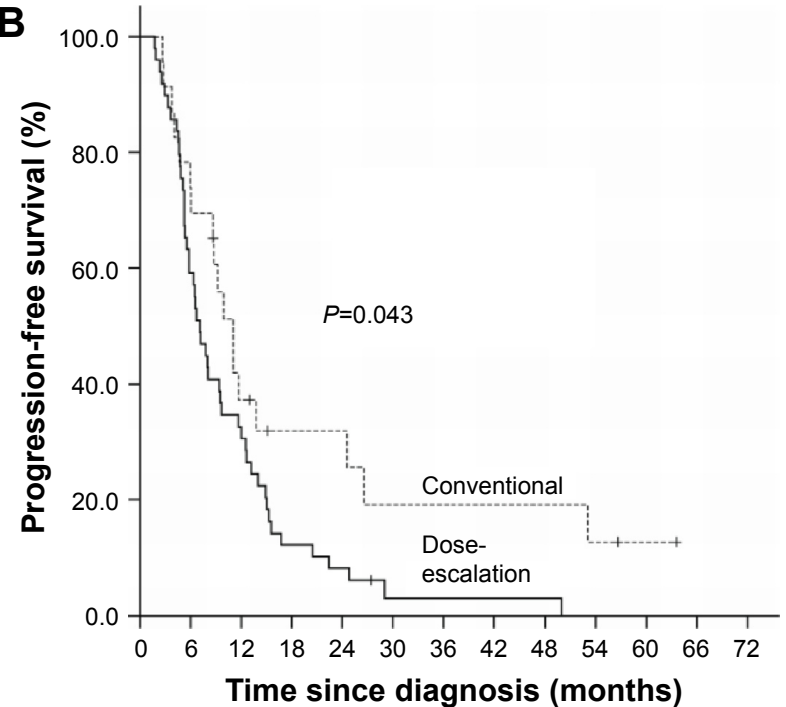

Number at risk:

$$
\text { Dose- }
$$

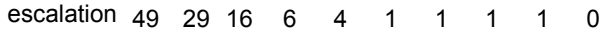

Conventional $\begin{array}{lllllllllllll}23 & 17 & 8 & 5 & 5 & 3 & 3 & 3 & 3 & 2 & 1 & 0\end{array}$

Figure 2 Kaplan-Meier estimates of both cohorts.

Notes: Kaplan-Meier estimates of overall survival (A) and progression-free survival (B)

(B) in dose-escalated (DE) and conventional radiotherapy cohorts.

whereas 32 relapsed (97\%) in the central area, and none in-field among the DE cohort $(P=0.019)$ (Table 2). Within the 70 Gy isodose, we observed 23 (69.7\%) central/in-field relapses, and ten (30.3\%) marginal/distal relapses.

Next we examined if relapse patterns within this highdose region differed between SVZ-contacting (SVZ+) and non-SVZ-contacting (SVZ-) tumors. Eight out of 20 (40\%) SVZ+ tumors relapsed marginally/distally, compared to two out of 13 (15.4\%) for SVZ- tumors (Table 3).

\section{Implications of radiation dose to the SVZ}

In patients who received $\mathrm{DE}$ radiotherapy, mean radiation doses to the ipsilateral, contralateral, and composite SVZ were 60.6 Gy (range 33.4-69.8), 39.5 Gy (range 19.4-61.2), and 49.1 Gy (range 28.3-64.3), respectively. Higher doses to the ipsilateral SVZ were associated with near statistical significance to superior PFS (HR $=0.95 ; 95 \%$ $\mathrm{CI}=0.9-1.0 ; P=0.052)$, but not $\mathrm{OS}$ of patients $(\mathrm{HR}=1.03$; $95 \% \mathrm{CI}=0.97-1.10 ; P=0.352$ ). Dose to the contralateral and composite SVZ was not correlated with either PFS or OS.

We next examined the association between the dosevolume of ipsilateral SVZ irradiation and PFS and OS in the same cohort. To this end, we utilized the $\mathrm{V}_{50}$ parameter for analysis based on the work by Chen et al. ${ }^{16} \mathrm{Mean}_{50}$ of the ipsilateral SVZ was $84.7 \%$ (range 22.5-100). Patients who received 50 Gy to the entire ipsilateral $\mathrm{SVZ}\left(\mathrm{V}_{50}=100 \%\right)$ experienced a better PFS, and this approached near statistical significance $(\mathrm{HR}=0.52 ; 95 \% \mathrm{CI}=0.27-1.02 ; P=0.055)$

\begin{tabular}{|c|c|c|c|c|c|c|c|}
\hline & \multicolumn{2}{|l|}{ Total } & \multicolumn{2}{|c|}{$\begin{array}{l}\text { Dose-escalated } \\
\text { cohort }\end{array}$} & \multicolumn{2}{|c|}{$\begin{array}{l}\text { Conventional } \\
\text { cohort }\end{array}$} & \multirow[t]{2}{*}{$P$-value } \\
\hline & Number & $\%$ & Number & $\%$ & Number & $\%$ & \\
\hline Number of relapsed patients & 51 & & 34 & & 17 & & \\
\hline $\begin{array}{l}\text { Among patients with planning } \\
\text { computed tomography details^ }\end{array}$ & 50 & 100 & 33 & 100 & 17 & 100 & \\
\hline \multicolumn{8}{|l|}{ Relapse patterns ${ }^{\#}$} \\
\hline Central & 44 & 88.0 & 32 & 97.0 & 12 & 70.6 & 0.019 \\
\hline In-field & 1 & 2.0 & 0 & - & I & 5.9 & \\
\hline Marginal & 3 & 6.0 & 1 & 3.0 & 2 & 11.8 & \\
\hline Distal & 2 & 4.0 & 0 & - & 2 & 11.8 & \\
\hline
\end{tabular}

Table 2 Relapse pattern within $60 \mathrm{~Gy}$ isodose

Notes: 'Unable to retrieve treatment plan for one patient in the dose-escalated cohort. "Central: $\geq 95 \%-100 \%$ of recurrence volume within 60 Gy isodose; in-field: $\geq 80 \%-95 \%$; marginal: $\geq 20 \%-80 \%$; distal: $<20 \%$. 
Table 3 Relapse pattern within 70 Gy isodose for dose-escalated cohort

\begin{tabular}{|c|c|c|c|c|c|c|c|}
\hline & \multicolumn{2}{|l|}{ Total } & \multicolumn{2}{|l|}{ SVZ+ } & \multicolumn{2}{|l|}{ SVZ- } & \multirow[t]{2}{*}{$P$-value } \\
\hline & Number & $\%$ & Number & $\%$ & Number & $\%$ & \\
\hline Number of relapsed patients & 34 & & & & & & \\
\hline $\begin{array}{l}\text { Among patients with planning } \\
\text { computed tomography details^ }\end{array}$ & 33 & 100 & 20 & 100 & 13 & 100 & \\
\hline \multicolumn{8}{|c|}{ Relapse pattern within $70 \mathrm{~Gy}$ isodose } \\
\hline Central or in-field & 23 & 69.7 & 12 & 60 & 11 & 84.6 & 0.246 \\
\hline Marginal or distal & 10 & 30.3 & 8 & 40 & 2 & 15.4 & \\
\hline
\end{tabular}

Notes: SVZ+ represents SVZ-contacting tumors and SVZ- represents non SVZ-contacting tumors; ^unable to retrieve treatment plan for one patient; \#central: $\geq 95 \%-100 \%$ of recurrence volume within $70 \mathrm{~Gy}$ isodose; in-field: $\geq 80 \%-95 \%$; marginal: $\geq 20 \%-80 \%$; distal: $<20 \%$.

Abbreviation: SVZ, subventricular zones.

(Figure 3). There was no association between OS and this parameter $(\mathrm{HR}=0.76 ; 95 \% \mathrm{CI}=0.38-1.54 ; P=0.446$, Figure 3).

\section{Discussion}

Our study demonstrated that DE radiation to newly-diagnosed GBM patients using 70 Gy over 30 fractions did not yield any advantage in OS, with about a third of the DE cohort still exhibiting central relapses within the 70 Gy isodose. As expected, TMZ non-recipients and Eastern Cooperative Oncology Group performance status 2-3 were unfavorable factors affecting survival. Exploratory analyses of radiation dose to the SVZ indicated that dose to the ipsilateral SVZ and volume receiving 50 Gy were associated with near statistical significance with PFS. It would be suggestive on the basis of the study findings that treatment intensification through
DE is ineffective in patients with GBM. Rather, the SVZ seem to be a potential target consideration when planning radiotherapy for these patients.

The observed lack of effect with DE in the current study is consistent with several other reports that have also failed to demonstrate a dose-response in tumor control and survival beyond conventional dose-schedules in patients with GBM. ${ }^{17,18}$ With the exception of the study by Tsien et al that reported a median PFS and OS of 9 and 20.1 months, respectively, with DE radiotherapy (75 Gy delivered in 30 fractions), approximately half of the patients in that study still failed centrally. ${ }^{19}$

Of interest however, ${ }^{11} \mathrm{C}$-methionine positron emission tomography (MET-PET) was available in the study by Tsien et $\mathrm{al}^{19}$ as an adjunct imaging tool for comparison against MRI in delineating the extent of residual disease post-surgical

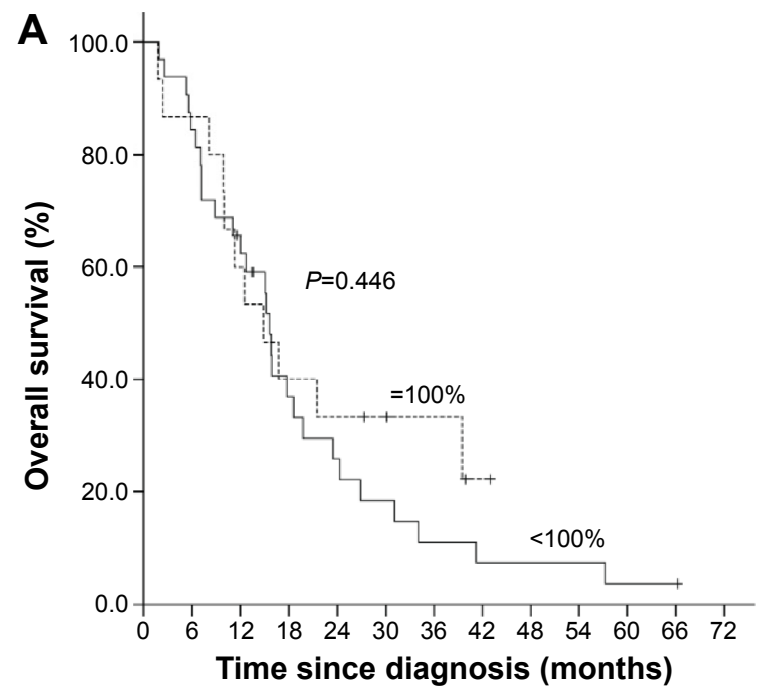

Number at risk:

$$
\begin{array}{lllllllllllll}
<100 \% & 32 & 27 & 20 & 10 & 7 & 5 & 3 & 2 & 2 & 2 & 1 & 1 \\
=100 \% & 15 & 13 & 9 & 6 & 5 & 4 & 3 & 1 & 0 & & &
\end{array}
$$

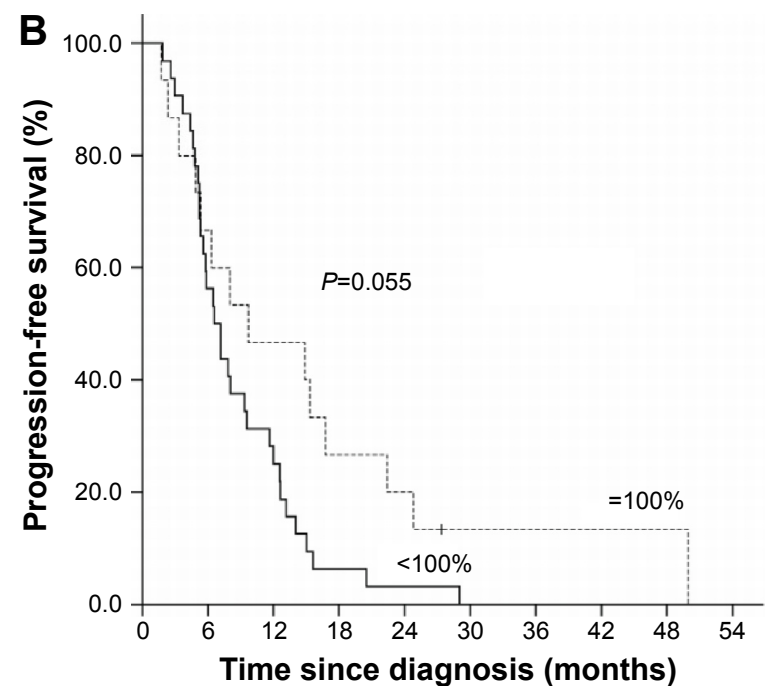

Number at risk:

$\begin{array}{llllllllll}<100 \% & 32 & 18 & 9 & 2 & 1 & 0 & & & \\ =100 \% & 15 & 10 & 7 & 4 & 3 & 1 & 1 & 1 & 1\end{array}$

Figure 3 Kaplan-Meier estimates based on volume of ipsilateral SVZ receiving 50 Gy.

Notes: Kaplan-Meier estimates of overall survival (A) and progression-free survival (B) based on volume of ipsilateral subventricular zones (SVZ) receiving 50 Gy $\left(\mathrm{V}_{50}\right)$ for patients treated with dose-escalated (DE) radiotherapy. 
resection (radiotherapy GTV). Although the defined volume of disease was much smaller with MET-PET, of particular significance was the observation that MET-PET identified areas of "tumor" that were not visible on MRI. As it stands, much needs to be done to validate the accuracy of PET-defined tumors, but it is reasonable to consider that limited success with DE trials could be attributed in part to the suboptimal efficacy of current imaging tools in defining "gross" tumor volume during the process of radiotherapy planning. ${ }^{20}$

A more recent study employing a unique scheme of high-dose hypofractionated radiotherapy also offered interesting insights into the radiation sensitivity of GBM cells. ${ }^{21}$ In addition to using an escalated dose of 68 Gy planned to a comparable "high dose" target volume, treatment was delivered over a course of eight fractions, which resulted in a fraction size of $8.5 \mathrm{~Gy}$ as opposed to $2.33 \mathrm{~Gy}$ in the current study. Preliminary survival analysis indicated promising median OS of 20 months in that cohort, and expectedly, 20 of 46 individuals developed cerebral necrosis post-therapy. Nonetheless, it was impressive to note that patterns of relapses differed in that local control was excellent, while distant failures predominated. Although preliminary, these findings certainly challenge the notion that GBM cells are insensitive to fraction size (high $\alpha / \beta$ ), in which case a hypofractionated approach may be more efficacious, as opposed to the timehonored method of conventional fractionation.

The clinical relevance of SVZ in patients with GBM has been repeatedly discussed in recent times, as evidenced by the number of studies that have proposed not only a prognostic association between the tumor and its anatomical relation to the SVZ, but also highlighting the potential impact of irradiating this structure within the brain parenchyma. ${ }^{16,22-24}$ It was first put forth by Lim et al that patients with GBM can be stratified into different prognostic risk categories depending on the presence of contact between the tumor and SVZ. ${ }^{11}$ Since then, the findings have been independently validated by two other groups, ${ }^{12,25}$ and it was further demonstrated that irradiation of the SVZ could influence PFS and OS of GBM patients. ${ }^{16,21,22,24}$ Among the DE cohort, we explored the hypothesis that the entire ipsilateral SVZ should be incorporated as a low-risk ( $50 \mathrm{~Gy}$ ) target volume for radiotherapy of GBM patients. We demonstrated that a higher radiation dose to the ipsilateral SVZ and the proportion of ipsilateral SVZ within the 50 Gy isodose resulted in a higher likelihood of improved PFS. Moreover, our exploratory analysis might suggest that relapse patterns differed between SVZ+ and SVZ- tumors, with marginal/distal relapses being more common in SVZ+ than SVZ- tumors. Collectively, these findings concur with a very recent report by Liang et al, ${ }^{26}$ and support our initiative to explore the clinical impact of radiation dose to the SVZ.

Nonetheless, our findings must be considered along with the limitations that this is a study undertaken retrospectively, examining the value of DE in GBM and the relationship of radiation dose to the SVZ with clinical outcomes. A flaw of the study, which is by no means insignificant, relates to the evident lack of centralized radiotherapy quality assurance. To partially account for this, a comprehensive review of the DE radiotherapy plans confirmed that 70 and 60 Gy encompassed at least $95 \%$ of planning target volume $(\mathrm{PTV})_{70}$ and $\mathrm{PTV}_{60}$, respectively, in all patients. It should also be mentioned that MGMT promoter methylation testing was not yet available during the study timeframe, and while this information is less crucial in influencing the clinical management of patients, it is important to acknowledge that this biomarker status could have confounded our study findings. Lastly, we acknowledge the fact that radiation necrosis as a consequence of DE was not formally evaluated in this retrospective study. While it is possible that this could have confounded our analysis of tumor recurrence following DE, diagnosing radiation necrosis remains a clinical challenge, with only few imaging techniques proven to be useful in this regard.

\section{Conclusion}

In conclusion, we failed to demonstrate that moderatelyhypofractionated DE above 60 Gy improves survival in GBM patients, with central failures constituting the majority of recurrences following definitive local therapy. Incorporation of ipsilateral SVZ as a radiotherapy target volume for GBM patients requires further validation.

\section{Acknowledgment}

Melvin Lee Kiang Chua is kindly supported by the National Medical Research Council Singapore Transition Award (NMRC/TA/0030/2014).

\section{Disclosure}

The authors report no conflicts of interest in this work.

\section{References}

1. Hegi ME, Diserens AC, Godard S, et al. Clinical trial substantiates the predictive value of O-6-methylguanine-DNA methyltransferase promoter methylation in glioblastoma patients treated with temozolomide. Clin Cancer Res. 2004;10(6):1871-1874.

2. Hegi ME, Diserens AC, Gorlia T, et al. MGMT gene silencing and benefit from temozolomide in glioblastoma. $N$ Engl J Med. 2005; 352(10):997-1003. 
3. Stupp R, Hegi ME, Gorlia T, et al. Cilengitide combined with standard treatment for patients with newly diagnosed glioblastoma with methylated MGMT promoter (CENTRIC EORTC 26071-22072 study): a multicentre, randomised, open-label, phase 3 trial. Lancet Oncol. 2014;15(10):1100-1108.

4. Chinot OL, Wick W, Mason W, et al. Bevacizumab plus radiotherapytemozolomide for newly diagnosed glioblastoma. $N \mathrm{Engl} \mathrm{J} \mathrm{Med.}$ 2014;370(8):709-722.

5. Gilbert MR, Dignam JJ, Armstrong TS, et al. A randomized trial of bevacizumab for newly diagnosed glioblastoma. N Engl J Med. 2014; 370(8):699-708.

6. Ma DJ, Galanis E, Anderson SK, et al. A phase II trial of everolimus, temozolomide, and radiotherapy in patients with newly diagnosed glioblastoma: NCCTG N057K. Neuro Oncol. 2015;17(9):1261-1269.

7. Sarkaria JN, Galanis E, Wu W, et al; North Central Cancer Treatment Group Phase I trial N057K of everolimus (RAD001) and temozolomide in combination with radiation therapy in patients with newly diagnosed glioblastoma multiforme. Int J Radiat Oncol Biol Phys. 2011;81(2): 468-475.

8. Sherriff J, Tamangani J, Senthil L, et al. Patterns of relapse in glioblastoma multiforme following concomitant chemoradiotherapy with temozolomide. Br J Radiol. 2013;86(1022):20120414.

9. Bao S, Wu Q, McLendon RE, et al. Glioma stem cells promote radioresistance by preferential activation of the DNA damage response. Nature. 2006;444(7120):756-760.

10. Tabatabai G, Frank B, Mohle R, Weller M, Wick W. Irradiation and hypoxia promote homing of haematopoietic progenitor cells towards gliomas by TGF-beta-dependent HIF-1alpha-mediated induction of CXCL12. Brain. 2006;129(Pt 9):2426-2435.

11. Lim DA, Cha S, Mayo MC, et al. Relationship of glioblastoma multiforme to neural stem cell regions predicts invasive and multifocal tumor phenotype. Neuro Oncol. 2007;9(4):424-429.

12. Jafri NF, Clarke JL, Weinberg V, Barani IJ, Cha S. Relationship of glioblastoma multiforme to the subventricular zone is associated with survival. Neuro Oncol. 2013;15(1):91-96.

13. Stupp R, Mason WP, van den Bent MJ, et al. Radiotherapy plus concomitant and adjuvant temozolomide for glioblastoma. $N$ Engl J Med. 2005;352(10):987-996.

14. Wen PY, Macdonald DR, Reardon DA, et al. Updated response assessment criteria for high-grade gliomas: response assessment in neurooncology working group. J Clin Oncol. 2010;28(11):1963-1972.
15. Barani IJ, Cuttino LW, Benedict SH, et al. Neural stem cell-preserving external-beam radiotherapy of central nervous system malignancies. Int J Radiat Oncol Biol Phys. 2007;68(4):978-985.

16. Chen L, Guerrero-Cazares H, Ye X, et al. Increased subventricular zone radiation dose correlates with survival in glioblastoma patients after gross total resection. Int J Radiat Oncol Biol Phys. 2013;86(4):616-622.

17. Jastaniyah N, Murtha A, Pervez N, et al. Phase I study of hypofractionated intensity modulated radiation therapy with concurrent and adjuvant temozolomide in patients with glioblastoma multiforme. Radiat Oncol. 2013;8:38.

18. Badiyan SN, Markovina S, Simpson JR, et al. Radiation therapy dose escalation for glioblastoma multiforme in the era of temozolomide. Int J Radiat Oncol Biol Phys. 2014;90(4):877-885.

19. Tsien CI, Brown D, Normolle D, et al. Concurrent temozolomide and dose-escalated intensity-modulated radiation therapy in newly diagnosed glioblastoma. Clin Cancer Res. 2012;18(1):273-279.

20. Miwa K, Shinoda J, Yano H, et al. Discrepancy between lesion distributions on methionine PET and MR images in patients with glioblastoma multiforme: insight from a PET and MR fusion image study. J Neurol Neurosurg Psychiatry. 2004;75(10):1457-1462.

21. Iuchi T, Hatano K, Kodama T, et al. Phase 2 trial of hypofractionated high-dose intensity modulated radiation therapy with concurrent and adjuvant temozolomide for newly diagnosed glioblastoma. Int J Radiat Oncol Biol Phys. 2014;88(4):793-800.

22. Evers P, Lee PP, DeMarco J, et al. Irradiation of the potential cancer stem cell niches in the adult brain improves progression-free survival of patients with malignant glioma. BMC Cancer. 2010;10:384.

23. Kut C, Janson Redmond K. New considerations in radiation treatment planning for brain tumors: neural progenitor cell-containing niches. Semin Radiat Oncol. 2014;24(4):265-272.

24. Lee P, Eppinga W, Lagerwaard F, et al. Evaluation of high ipsilateral subventricular zone radiation therapy dose in glioblastoma: a pooled analysis. Int J Radiat Oncol Biol Phys. 2013;86(4):609-615.

25. Adeberg S, Konig L, Bostel T, et al. Glioblastoma recurrence patterns after radiation therapy with regard to the subventricular zone. Int J Radiat Oncol Biol Phys. 2014;90(4):886-893.

26. Liang TH, Kuo SH, Wang CW, et al. Adverse prognosis and distinct progression patterns after concurrent chemoradiotherapy for glioblastoma with synchronous subventricular zone and corpus callosum invasion. Radiother Oncol. Epub 2015 Dec 8.
OncoTargets and Therapy

\section{Publish your work in this journal}

OncoTargets and Therapy is an international, peer-reviewed, open access journal focusing on the pathological basis of all cancers, potential targets for therapy and treatment protocols employed to improve the management of cancer patients. The journal also focuses on the impact of management programs and new therapeutic agents and protocols on

\section{Dovepress}

patient perspectives such as quality of life, adherence and satisfaction The manuscript management system is completely online and includes a very quick and fair peer-review system, which is all easy to use. Visit http://www.dovepress.com/testimonials.php to read real quotes from published authors. 